\title{
PRIMARY DECOMPOSITION OF A 0-CONNECTED NILPOTENT SPACE
}

Akrur Behera and Sandhya Rani Mohapatra

Department of Mathematics, National Institute of Technology, Rourkela Rourkela - 769008 (India)

Department of Mathematics, National Institute of Technology, Rourkela Rourkela - 769008 (India)

\section{ABSTRACT}

It is well known that the different stages of the Cartan-Whitehead decomposition of a 0-connected space can be obtained as the Adams cocompletion of the space with respect to suitable sets of morphisms. In thispaper Cartan-Whitehead decomposition is obtained for a nilpotent space, in terms of Adams cocompletion, using the primary homotopy theory developed by Neisendonfer.

\section{Indexing terms/Keywords}

Category of fractions; Calculus of right fractions; Adams cocompletion; Primary homotopy theory; Nilpotent space; CartanWhitehead decomposition.

\section{Academic Discipline And Sub-Disciplines}

Mathematics, Category Theory, Algebraic Topology.

\section{SUBJECT CLASSIFICATION}

AMS : 18A35, 55P60

\section{TYPE (METHOD/APPROACH)}

A new approach to Cartan-Whitehead decomposition of a nilpotent space.

\section{Council for Innovative Research}

Peer Review Research Publishing System

Journal: JOURNAL OF ADVANCES IN MATHEMATICS

Vol.11, No.7

www.cirjam.com , editorjam@gmail.com 


\section{INTRODUCTION}

Deleanu, Frei and Hilton have developed the notion of generalized Adams completion in a categorical context; they have also studied the dual notion, namely the Adams cocompletion of an object in a category [9]. It is to be emphasized that many algebraic and geometrical constructions in Algebraic Topology, Differential Topology, Differentiable Manifolds, Algebra, Analysis, Topology, etc., can be viewed as Adams completions or cocompletions of objects in suitable categories, with respect to carefully chosen sets of morphisms. Behera and Nanda [3] have shown that the different stages of the Cartan-Whitehead decomposition of a 0 -connected space are the Adams cocompletion of a space with respect to suitable sets of morphisms. In [12], Neisendonfer has studied the primary homotopic theory in an exhaustive manner.. The central idea of this note is to study how Cartan-Whitehead decomposition of a 0-connected nilpotent space is characterized in terms of its Adams cocompletions; it is done using the primary homotopy theory developed by Neisendonfer.

Let $\mathcal{C}$ be an arbitrary category and $\mathrm{S}$ a set of morphisms of $\mathcal{C}$. Let $\mathcal{C}\left[\mathrm{S}^{-1}\right]$ denote the category of fractions of $\mathcal{C}$ with respectS andF: $\mathcal{C} \rightarrow \mathcal{C}\left[\mathrm{S}^{-1}\right]$ be the canonical functor. Let $\mathcal{S}$ denote the category of sets and functions. Then for a given object $\mathrm{Y}$ of $\mathcal{C}$,

$$
\mathcal{C}\left[\mathrm{S}^{-1}\right](\mathrm{Y},-): \mathcal{C} \rightarrow \mathcal{S}
$$

defines a covariant functor. If this functor is representable by an object $\mathrm{Y}_{\mathrm{S}}$ of $\mathcal{C}$, that is, if

$$
\mathcal{C}\left[\mathrm{S}^{-1}\right](\mathrm{Y},-) \cong \mathcal{C}\left(\mathrm{Y}_{\mathrm{S}},-\right)
$$

then $Y_{S}$ is called the (generalized) Adams cocompletion of $Y$ with respect to the set of morphisms $S$ or simply the $S$ cocompletion ofY. We shall often refer to $\mathrm{Y}_{\mathrm{S}}$ as the cocompletion of $\mathrm{Y}$ [9].

Given aset $\mathrm{S}$ of morphisms of $\mathcal{C}$, the saturation of $\mathrm{S}$, denoted as $\overline{\mathrm{S}}$ is the set of all morphisms $\mathrm{u}$ in $\mathcal{C}$ such that $\mathrm{F}(\mathrm{u})$ is an isomorphism in $\mathcal{C}\left[\mathrm{S}^{-1}\right]$. Furthermore, $\mathrm{S}$ is said to be saturated if $\mathrm{S}=\overline{\mathrm{S}}[4,9]$.

Deleanu, Frei and Hilton have shown that if the set of morphisms Sis saturated then the Adams cocompletion of a space is characterized by a certain couniversal property ([9],dual of Theorem 1.2). In most of the applications, however, the set of morphisms $\mathrm{S}$ is not saturated. There is a stronger version of Deleanu, Frei and Hilton's characterization of Adams cocompletion in terms of couniversal property as described below.

Theorem1.1. ([4], dual of Theorem 1.2) Let $\mathrm{S}$ be a set of morphisms of $\quad \mathcal{C}$ admitting a calculus of right fractions. Then an object $\mathrm{Y}_{\mathrm{S}}$ of $\mathcal{C}$ is the $\mathrm{S}$-cocompletion of the object $\mathrm{Y}$ with respect to $\mathrm{S}$ if and only if there exists a morphism $\mathrm{e}: \mathrm{Y}_{\mathrm{S}} \rightarrow \mathrm{Y}$ in $\overline{\mathrm{S}}$ which is couniversal with respect to morphisms ins : given a morphism $\mathrm{S}: \mathrm{Z} \rightarrow \mathrm{Y}$ in $\mathrm{S}$ there exists a unique morphism $t: Y_{S} \rightarrow Z$ in $\bar{S}$ such that $s t=e$. In other words, the following diagram is commutative :

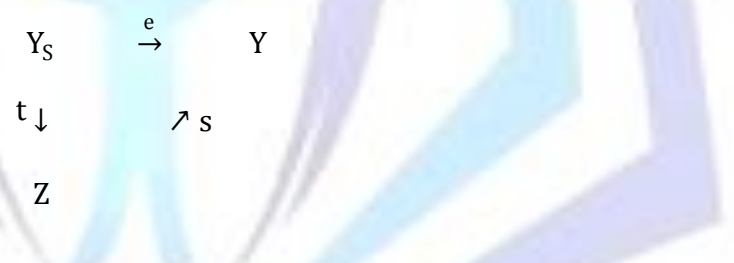

Also the above theorem turns out to be essentially the dual of Theorem 1.2 [9] if we assume $S$ to be saturated; hence the proposition can be proved by recasting the dual of the proof of the Theorem 1.2 [9] with minor changes. The details are omitted.

The following Theorem (dual of Theorem 1.3, [4]) shows that under certain conditions the morphisms e: $\mathrm{Y}_{\mathrm{S}} \rightarrow \mathrm{Y}$ always belongs to $\mathrm{S}$.

Theorem1.2 . ([4], dual of Theorem 1.3) Let $S=S_{1} \cap S_{2}$. be a set of morphisms in a category $\mathcal{C}$ admitting a calculus of right fractions. Let $\mathrm{e}: \mathrm{Y}_{\mathrm{S}} \rightarrow \mathrm{Y}$ be the canonical morphism as defined in Theorem 1.1, where $\mathrm{Y}_{\mathrm{S}}$ is the $\mathrm{S}$ cocompletion of $\mathrm{Y}$. Assume further that $\mathrm{S}_{1}$ and $\mathrm{S}_{2}$ have the following properties :

(i) $\quad \mathrm{S}_{1}$ and $\mathrm{S}_{2}$ are closed under composition.

(ii) $\quad$ fg $\in \mathrm{S}_{1}$ implies thatg $\in \mathrm{S}_{1}$.

(iii) $\quad$ fg $\in S_{2}$ implies thatf $\in S_{2}$.

Then $\mathrm{e} \in \mathrm{S}$.

\section{The category $\mathcal{N}_{0}$.}

Let $\mathrm{S}^{\mathrm{m}}$ denote the m-dimensional sphere. Suppose $\mathrm{m} \geq 2$, and let $\mathrm{k}: \mathrm{S}^{\mathrm{m}} \rightarrow \mathrm{S}^{\mathrm{m}}$ denote a map of degree $\mathrm{k}$. The space $S^{m-1} U_{k} e^{m}$ is denoted by $\mathrm{P}^{\mathrm{m}}(\mathrm{k})$ or $\mathrm{P}^{\mathrm{m}}(\mathbb{Z} / \mathrm{k} \mathbb{Z})$. If $\mathrm{m} \geq 2$, the $\mathrm{m}$-th modkhomotopy group of $\mathrm{X}$ is $\left[\mathrm{P}^{\mathrm{m}}(\mathrm{k}) ; \mathrm{X}\right]$, denoted by $\pi_{m}(X ; \mathbb{Z} / k \mathbb{Z})$ [12]. If $m \geq 3, \quad \pi_{m}(X ; \mathbb{Z} / k \mathbb{Z})$ is a group and $m \geq 4, \quad \pi_{m}(X ; \mathbb{Z} / k \mathbb{Z})$ is an abelian group [12]. 
If $\mathrm{f}: \mathrm{X} \rightarrow \mathrm{Y}$ is a map, then there are induced maps $\mathrm{f}_{*}: \pi_{\mathrm{m}}(\mathrm{X} ; \mathbb{Z} / \mathrm{kZ}) \rightarrow \pi_{\mathrm{m}}(\mathrm{Y} ; \mathbb{Z} / \mathrm{kZ})$ defined by $\mathrm{f}_{*}[\mathrm{~g}]=[\mathrm{fg}]$. If $\mathrm{m} \geq 3, \mathrm{f}_{*}$ is a homomorphism and, if $\mathrm{m}=2, \mathrm{f}_{*}$ is compatible with the action of $\pi_{2}[12]$.

For a group G, the lower central series

$$
\cdots \subseteq \Gamma^{\mathrm{i}+1}(\mathrm{G}) \subseteq \Gamma^{\mathrm{i}}(\mathrm{G}) \cdots \subseteq \Gamma^{1}(\mathrm{G})
$$

of $\mathrm{G}, \quad$ is defined by the setting

$$
\Gamma^{1}(\mathrm{G})=\mathrm{G}, \quad \Gamma^{\mathrm{i}+1}(\mathrm{G})=\left[\mathrm{G}, \Gamma^{\mathrm{i}}(\mathrm{G})\right], \quad \mathrm{i} \geq 1 .
$$

$\mathrm{G}$ is said to be nilpotent if $\Gamma^{j}(G)=\{1\}$ for $j$ sufficiently large [10].

A connected $C W$-complex $X$ is said to nilpotent if $\pi_{1}(X)$ is nilpotent and operates nilpotently on $\pi_{n}(X)$ for every $n \geq 2[10]$.

Let $\quad \mathcal{N}_{0}$ denote the category of 0 -connected based nilpotent spaces and based maps and let $\widetilde{\mathcal{N}}_{0}$ be the corresponding homotopy category. We assume that the underlying sets of the elements of $\tilde{\mathcal{N}}_{0}$ are the elements of $u$, where $U$ is a fixed Grothendeick universe. We now fix suitable sets of morphisms of $\widetilde{\mathcal{N}}_{0}$.

Let $S_{n}$ denote the set of all maps $\alpha$ in $\widetilde{\mathcal{N}}_{0}$ having the following property: $\alpha: X \rightarrow Y$ is in $S_{n}$ if and only if $\alpha_{*}$ : $\pi_{m}(X ; \mathbb{Z} / k \mathbb{Z}) \rightarrow \pi_{m}(Y ; \mathbb{Z} / k \mathbb{Z})$ is an isomorphism for $m>n$ and a monomorphism for $m=n$.

Proposition 2.1. $S_{n}$ admits a calculus of right fractions.

Proof. Clearly $S_{n}$ is closed under composition. We shall verify conditions (i) and (ii) of Theorem 1.3* [9]. Only condition (ii) is in question. For proving this condition it is enough to prove that every diagram

in $\widetilde{\mathcal{N}}_{0}$ with $\gamma \in S_{n}$, can be embedded in a weak pull-back diagram
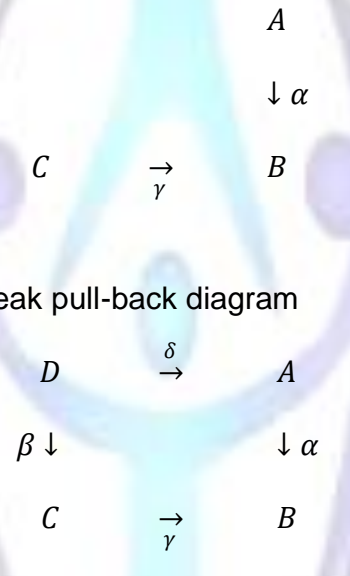

with $\delta \in S_{n}$. Suppose $\alpha=[f]$ and $\gamma=[s]$. We replace $f$ and $s$ by fibrations $f^{\prime}$ and $s^{\prime}$ respectively; we have $f=f^{\prime} r: A \stackrel{r}{\rightarrow} P_{f} \stackrel{f^{\prime}}{\rightarrow} B$ and $s=s^{\prime} t: C \stackrel{t}{\rightarrow} P_{s} \stackrel{s^{\prime}}{\rightarrow} B$ where $r$ and $t$ are homotopy equivalences and $P_{f} \quad$ and $P_{s}$ are mapping tracks of $f$ and $s$ respectively. Let $\bar{r}$ and $\bar{t}$ be the homotopy inverses of $r$ and $t$ respectively. Let $D$ be the usual pull-back of $f^{\prime}$ and $s^{\prime}$ and $p: D \rightarrow P_{f}, \quad q: D \rightarrow P_{s}$ be the respective projections. Let $\delta=[\bar{r} p] \quad$ and $\beta=[\bar{t} q]$. Thus $\alpha \delta=[f][\bar{r} p]=[f \bar{r} p]=\left[f^{\prime} r \bar{r} p\right]=\left[f^{\prime} p\right]=\left[s^{\prime} q\right]=\left[s^{\prime} t \bar{t} q\right]=[s \bar{t} q]=[s][\bar{t} q]=\gamma \beta$. Moreover, if $\alpha \mu=\gamma \lambda$, let $u: U \rightarrow A, \quad v: U \rightarrow C$ be in the classes $\mu, \lambda$ respectively so that $f u \simeq s v$ or $f^{\prime} r u \simeq s v$. Let $F: U \times I \rightarrow B$ be a homotopy with $F_{0}=f^{\prime} r u$ and $F_{1}=s v$. Since $f^{\prime}$ is a fibration there exists a homotopy $G: U \times I \rightarrow$ $P_{f}$ such that $f^{\prime} G_{t}=F_{t}$ and $G_{0}=r u$. Thus $f^{\prime} G_{1}=F_{1}=s v=s^{\prime} t v$. By the pull-back property of $D$ there exists a map $k: U \rightarrow D$ such that $p k=G_{1} \simeq r u$ and $q k=t v$. Thus if $\rho=[k]$, then $\delta \rho=[\bar{r} p][k]=[\bar{r} p k]=[\bar{r} r u]=[u]=\mu$ and $\beta \rho=[\bar{t} q][k]=[\bar{t} q k]=[\bar{t} t v]=[v]=\lambda$.

It remains to be shown that $\delta \in S_{n}$. We assume that the map $\alpha: A \rightarrow B$ is a fibration with fibre $Q$. We note that $Q$ is also the fibre of $\beta: D \rightarrow C$. We have the following commutative diagram.

$$
\begin{aligned}
& \cdots \rightarrow \pi_{m+1}(C ; \mathbb{Z} / k \mathbb{Z}) \rightarrow \pi_{m}(Q ; \mathbb{Z} / k \mathbb{Z}) \rightarrow \pi_{m}(D ; \mathbb{Z} / k \mathbb{Z}) \rightarrow \pi_{m}(C ; \mathbb{Z} / k \mathbb{Z}) \rightarrow \pi_{m-1}(Q ; \mathbb{Z} / k \mathbb{Z}) \rightarrow \cdots \\
& \begin{array}{clcl}
\gamma_{*} \downarrow & \| & \delta_{*} \downarrow & \gamma_{*} \downarrow
\end{array} \\
& \cdots \rightarrow \pi_{m+1}(B ; \mathbb{Z} / k \mathbb{Z}) \rightarrow \pi_{m}(Q ; \mathbb{Z} / k \mathbb{Z}) \rightarrow \pi_{m}(A ; \mathbb{Z} / k \mathbb{Z}) \rightarrow \pi_{m}(B ; \mathbb{Z} / k \mathbb{Z}) \rightarrow \pi_{m-1}(Q ; \mathbb{Z} / k \mathbb{Z}) \rightarrow \cdots
\end{aligned}
$$

By Five Lemma $\quad \delta_{*}$ is an isomorphism for $m>n$ and a monomorphism for $m=n$, showing $\delta \in S_{n}$. This completes the proof of Proposition 2.1.

In fact, the set $S_{n}$ admits astrong calculus of right fractions. A set $S$ of morphisms of a small $\mathcal{V}$-category $\mathcal{C}, \mathcal{V}$ being a Grothendieck universe, admits a strong calculus of right fractions[14] if

(i) $S$ admits a calculus of right fractions, 
(ii) for any set $\left\{s_{i}: B_{i} \rightarrow A, i \in I, I\right.$ is a $\mathcal{V}$-set $\}$, there exists a commutative completion $\left\{f_{i}: C \rightarrow B_{i}, i \in I\right\} \quad$ such that $s_{i} f_{i} \in S$ for every $i \in I$.

Proposition 2.2. $S_{n}$ admits a strong calculus of right fractions.

Proof. Let $\left\{s_{i}: Y_{i} \rightarrow X, i \in I\right\}$ be a given set of morphisms in $\widetilde{\mathcal{N}}_{0}$ with every $s_{i} \in S_{n}$ and $I \in U$. We have a map from $X \rightarrow P^{n} X$, where $P^{n} X$ denotes the Postnikov decomposition of $X$ ([10], proof of Proposition 1.1). Convert this into a fibration: $X_{n} \stackrel{u_{n}}{\rightarrow} X \rightarrow P^{n} X, X_{n}$ being its fibre. Considering the homotopy exact sequence of this fibration we get $\pi_{m}\left(X_{n} ; \mathbb{Z} / k \mathbb{Z}\right)=0$ for $m \leq n$ and $\pi_{m}\left(X_{n} ; \mathbb{Z} / k \mathbb{Z}\right) \cong \pi_{m}(X ; \mathbb{Z} / k \mathbb{Z})$ for $m>n$. Thus $u_{n} \in S_{n}$. Since $\pi_{1}\left(X_{n} ; \mathbb{Z} / k \mathbb{Z}\right)=0$ we have a map (lifting) $f_{i}: X_{n} \rightarrow Y_{i}$ such that $s_{i} f_{i}=u_{n}$ and the proposition is proved.

Remark 2.3. We note that the map $u_{n}: X_{n} \rightarrow X$ is independent of the indexi.

Proposition 2.4. For a given object $X$ of the category $\widetilde{\mathcal{N}}_{0}$, there exists asubset $S_{X}$ of the $\operatorname{set}\left\{s: X^{\prime} \rightarrow X \mid s \in\right.$ $S_{n}$ \}such that $S_{X}$ is an element of theuniverse Uand for each $s: X^{\prime} \rightarrow X, s \in S_{n}$, there exists ans $s^{\prime}: X^{\prime \prime} \rightarrow X$ in $S_{X}$ and a morphism $u: X^{\prime \prime} \rightarrow X^{\prime}$ in $\widetilde{\mathcal{N}}_{0}$ such that $s u=s^{\prime}$.

Proof. For a given object $X$ in $\widetilde{\mathcal{N}}_{0}$, let $S_{X}$ denote the set of morphisms $S_{X}=\left\{s: Y \rightarrow X \mid s \in S_{n}, Y\right.$ is an object of $\left.\tilde{\mathcal{N}}_{0}\right\}$. We assert that $S_{X}$ is an element of $\mathcal{U}$. For any object $Y$ of $\widetilde{\mathcal{N}}_{0}$, let $S_{Y, X}=\left\{s: Y \rightarrow X, s \in S_{n}\right\}$. It is clear that $S_{X}=\cup_{Y} S_{Y, X}$ and $S_{Y, X}=S_{n} \cap \operatorname{Mor}_{\widetilde{N}_{0}}(Y, X)$. Since $\widetilde{\mathcal{N}}_{0}$ is a small $U$-category, $\operatorname{Mor}_{\widetilde{\mathcal{N}}_{0}}(Y, X)$ belongs to $\widetilde{\mathcal{N}}_{0}$ and so does $S_{Y, X}$, being a subset of $\operatorname{Mor}_{\widetilde{N}_{0}}(Y, X)$. Therefore, the set $S_{X}$, being a union of sets all belonging to $u$ and indexed by the objects $Y$ of $\widetilde{\mathcal{N}}_{0}$ (which is a subset of $U$ ) is itself in $U$.In view of Proposition 2.2 and Remark 2.3, there exists a lifting $f_{s}: X_{n} \rightarrow Y$ of $u$ such that $s f_{s}=u_{n}$ where $s \in S_{X}$ is arbitrary, $u_{n}$ is the map as constructed in Proposition 2.2. This completes the proof of the Proposition 2.4.

Corollary 2.5. $u_{n} \in S_{n}$ and with respect to anys $\in S_{X}, \quad u_{n}$ has couniversal property.

\section{Existence of Adams cocompletion in $\widetilde{\mathcal{N}}_{0}$.}

Since the category $\widetilde{\mathcal{N}}_{0}$ as stated above is neither complete nor small, the dual of Theorem 2.6 [7] nan not be used to show the existence of Adams cocompletion of an object in the category $\widetilde{\mathcal{N}}_{0}$ with respect to the set of morphisms $S_{n}$. The following theorem shows that under certain conditions the Adams cocompletion of an object in the category $\widetilde{\mathcal{N}}_{0}$ always exists; the theorem is essentially the dual of Theorem 4.7 [1] and dual of Theorem 3.8 [2] (it is also a generalization of the dual of the Theorem in [7]).

Theorem 3.1.Let $u$ be a fixed Grothendieck universe. Let $\tilde{\mathcal{C}}$ be the category defined as follows : the objects of $\tilde{\mathcal{C}}$ are connected based nilpotent spaces whose underlying sets are elements of $\mathcal{U}$; the morphisms of $\tilde{\mathcal{C}}$ are based homotopy classes of based-point preserving maps between such based nilpotent spaces. Let $S$ be a family of morphisms of $\tilde{\mathcal{C}}$ admitting a calculus of right fractions and satisfying the following axioms of compatibility with products :

(P) If $s_{i}: X_{i} \rightarrow Y_{i}$ lies in $S$ for each $i \in I$, where the index set $I$ is an element of $U$, then

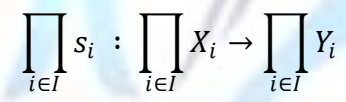

lies in $S$.

Assume that the family $S$ and the object $X$ of $\tilde{\mathcal{C}}$ satisfy the condition :

$(*)$ There exists a subset $S_{X}$ of the set $\left\{s: X^{\prime} \rightarrow X \mid s \in S\right\}$ such that $S_{X}$ is an element of the universe $u$ and for each $s: X^{\prime} \rightarrow X, s \in S$, there exist an $s^{\prime}: X^{\prime \prime} \rightarrow X$ in $S_{X}$ and a morphism $u: X^{\prime \prime} \rightarrow X^{\prime}$ of $\tilde{\mathcal{C}}$ rendering the following diagram is commutative :

$\begin{array}{lll}X^{\prime} & \stackrel{s}{\rightarrow} & X \\ u \uparrow & \nearrow s^{\prime} & \\ X^{\prime \prime} & & \end{array}$

Then the Adams cocompletion $X_{S}$ of $X$ does exist.

As remarked by Adams of page 34 of [2] this result remains valid if $\tilde{\mathcal{C}}$ is the homotopy category of 0 -connected based nilpotent spaces (whose underlying sets belong to $\mathcal{U}$ ). It is to be emphasized that condition $(*)$ is essential in order to be able to apply E.H. Brown's representability theorem to prove this result.

From the Propositions 2.1, 2.2 and 2.4 and Remark2.3, we note that the conditions of Theorem 3.1 are satisfied and so by Theorem 1.2, we obtain the following theorem.

Theorem 3.2. Every object $X$ of the category $\widetilde{\mathcal{N}}_{0}$ has an Adams cocompletion $X_{S_{n}}$ with respect to the set of morphisms $S_{n}$ and there exists a morphisme $e_{n}: X_{S_{n}} \rightarrow X$ in $\bar{S}_{n}$ which is couniversal with respect to morphisms in $S_{n}$. 
Proposition 3.3. The morphisme $e_{n}: X_{S_{n}} \rightarrow X$ as constructed in Theorem3.2is in $S_{n}$.

Proof. The proof follows from the dual of Theorem 1.3 [4]. We take $S_{n}^{1}=\left\{\alpha: X \rightarrow Y\right.$ in $\widetilde{\mathcal{N}_{0}} \mid \alpha_{*}: \pi_{m}(X ; \mathbb{Z} / k \mathbb{Z}) \rightarrow$ $\pi_{m}(Y ; \mathbb{Z} / k \mathbb{Z})$ is a monomorphism for $\left.m \geq n\right\}$ and $S_{n}^{2}=\left\{\alpha: X \rightarrow Y\right.$ in $\widetilde{\mathcal{N}}_{0} \mid \alpha_{*}: \pi_{m}(X ; \mathbb{Z} / k \mathbb{Z}) \rightarrow \pi_{m}(Y ; \mathbb{Z} / k \mathbb{Z})$ is an epimorphism for $m \geq n+1\}$. Clearly (a) $S_{n}=S_{n}^{1} \cap S_{n}^{2}$ and (b) $S_{n}^{1}$ and $S_{n}^{2}$ satisfy all conditions of Theorem 1.2; hence $e \in S_{n}$. This completes the proof of the Proposition 3.3.

\section{A primary decomposition of a 0 -connected based nilpotent space.}

Now we obtain the primary decomposition of a 0-connected based nilpotent space with the help of the set of morphisms $S_{n}$ as described below. In fact the different stages of the Cartan-Whitehead decomposition of a 0 -connected nilpotent space are the Adams cocompletions of the space with respect to the sets of morphisms $S_{n}$. In the process, starting from a 0 -connected based nilpotent space $X$ we get a tower of spaces,

$$
\cdots \rightarrow X_{n+1} \stackrel{\theta_{n+1}}{\rightarrow} X_{n} \rightarrow \cdots \rightarrow X_{1} \stackrel{\theta_{1}}{\rightarrow} X_{0}
$$

and the direct limit of this tower gives us a space which in some sense is the Cartan-Whitehead decomposition of $X$. First we prove the following proposition.

Proposition 4.1. $X_{n}$, as constructed in the proof of Proposition 2.2, is homotopically equivalent to $X_{S_{n}}$, as constructed in Theorem3.2.

Proof. By the couniversal property of $u_{n}: X_{n} \rightarrow X$ we have a map $s: X_{n} \rightarrow X_{S_{n}}$ such that $e_{n} s=u_{n}$. By the couniversal property of $e_{n}: X_{S_{n}} \rightarrow X$ we have a map $t: X_{S_{n}} \rightarrow X_{n}$ such that $u_{n} t=e_{n}$. Thus $u_{n}=e_{n} s=u_{n} t s i m p l i e s$ that $t s=1_{X_{n}}$ and $e_{n}=u_{n} t=e_{n} s t$ timplies that $s t=1_{X_{S_{n}}}$ and the required homeomorphism between $X_{n}$ and $X_{S_{n}}$ is obtained. This completes the proos of Proposition 4.1.

Theorem 4.2. Let $X$ be a 0 -connected based nilpotent space. Then for $n \geq 3$, there exist 0 -connected based nilpotent spaces $X_{n}$, mapse $e_{n}: X_{n} \rightarrow X$ andfibrations $\theta_{n+1}: X_{n+1} \rightarrow X_{n}$ such that

(a) $\quad e_{n_{*}}: \pi_{m}\left(X_{n} ; \mathbb{Z} / \mathrm{k} \mathbb{Z}\right) \rightarrow \pi_{\mathrm{m}}(\mathrm{X} ; \mathbb{Z} / \mathrm{k} \mathbb{Z})$ is an isomorphism form $>n$ and $\pi_{\mathrm{m}}\left(\mathrm{X}_{\mathrm{n}} ; \mathbb{Z} / \mathrm{k} \mathbb{Z}\right)=0$ form $\leq \mathrm{n}$,

(b) $e_{n+1}=e_{n} \circ \theta_{n+1}$.

Proof. For each integer $n \geq 3$, let $X_{n}$ be the $S_{n}$-completion of $X$ and $e_{n}: X_{n} \rightarrow X$ be the canonical map as stated in Theorem 3.1. Since $e_{n} \in S_{n} \subset S_{n+1}$, it follows from the couniversal property of $e_{n+1}$ that there exists a unique morphism $\theta_{n+1}: X_{n+1} \rightarrow X_{n}$ such that $e_{n+1}=e_{n} \circ \theta_{n+1}$, i.e., the following diagram is commutative:

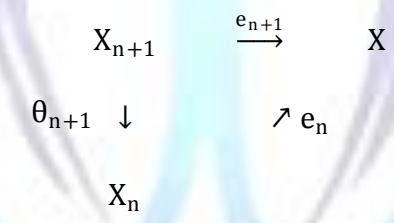

The maps $\theta_{\mathrm{n}}$ can of course be replaced by fibrations in the usual manner. Since $e_{\mathrm{n}} \in S_{\mathrm{n}}, \mathrm{e}_{\mathrm{n}_{*}}: \pi_{\mathrm{m}}\left(\mathrm{X}_{\mathrm{n}} ; \mathbb{Z} / \mathrm{kZ}\right) \rightarrow$ $\pi_{\mathrm{m}}(\mathrm{X} ; \mathbb{Z} / \mathrm{kZ})$ is an isomorphism for $\mathrm{m}>n$; it is already proved in Proposition 2.2 that $\pi_{\mathrm{m}}\left(\mathrm{X}_{\mathrm{n}} ; \mathbb{Z} / \mathrm{kZ}\right)=0$ for $\mathrm{m} \leq \mathrm{n}$.

This completes the proof of the Theorem 4.2.

\section{REFERENCES}

[1] Adams J.F. : Idempotent functors in homotopy theory; University of Chicago Press, 247 - 253 (1973).

[2] Adams J.F. : Localization and Completion; Lecture Notes in Mathematics, University of Chicago (1975).

[3] Behera A. and Nanda S. : Cartan-Whitehead decomposition as Adams cocompletion; J. Austral. Math. Soc.

(Series A) 42, 223 - 226, (1987).

[4] Behera A. and Nanda S. : Mod-C Postnikov Approximation of a1-connected space; Canad. J. Math. 39(3), 527 543, (1987).

[5] Bousfield A.K. : The localization of spaces with respect to homology; Topology 14, 133 - 150, (1975).

[6] Deleanu A.: Existence of the Adams completion for CW-complexes: J. Pure and Appl. Alg. 4, 299 - 308, (1974).

[7] Deleanu A. : Existence of the Adams completion for objects of cocomplete categories: J. Pure and Appl. Alg. 6, 31 - 39, (1975).

[8] Deleanu A. and Hilton P.J. : Localization, homology and a construction of Adams: Trans. Amer. Math. Soc., 179, 349 - 362, (1973). 
[9] Deleanu A., Frei A. and Hilton P.J. : Generalized Adamscompletion: Cahiers de Top. et Geom. Diff. 15, 61 82, (1974).

[10] Hilton P., Mislin G. and Roitberg J. : Localization of Nilpotent Groups and Spaces : North-Holland Mathematical Studies (1975).

[11] Nanda S. : A note on the universe of a category of fractions : Canad. Math. Bull., 23(4), 425 - 427, (1980).

[12] Neisendonfer J. : Primary Homotopy Theory : Memoirs AMS, 25 (232), (1980).

[13] SatyaDeo : Algebraic Topology - A Primer : Hindustan Book Agency, New Delhi (2003).

[14] Schubert H.: Categories: Springer-Verlag, New York (1972).

[15] Spanier E.H. : Algebraic Topology: McGraw-Hill Book Company, New York (1966).

[16] Switzer R.M.: Algebraic Topology - Homotopy and Homology : Springer-Verlag, Berlin (1975).

[17] Whitehead G. W. : Elements of Homotopy Theory : Springer-Verlag, New York (1978). 